\title{
Magnetic cycles at different ages of stars
}

\author{
K. Oláh ${ }^{1}$, Zs. Kôvári ${ }^{1}$, K. Petrovay ${ }^{2}$, W. Soon ${ }^{3}$, S. Baliunas ${ }^{4}$, Z. Kolláth ${ }^{5}$, and K. Vida ${ }^{1}$ \\ 1 Konkoly Observatory, Research Centre for Astronomy and Earth Sciences, Hungarian Academy of Sciences, 1121 Budapest, \\ Konkoly Thege M. u. 15-17, Hungary \\ e-mail: olah@konkoly.hu \\ 2 Eötvös University, Department of Astronomy, Pf. 32, 1518 Budapest, Hungary \\ 3 Harvard-Smithsonian Center for Astrophysics, Cambridge, MA 02138, USA \\ 4 c/o Dr. W. Soon, Harvard-Smithsonian Center for Astrophysics, Cambridge, MA 02138, USA \\ 5 University of West Hungary, Savaria Campus, Institute of Mathematics and Physics, Sopron 9400, Hungary
}

Received 10 March 2016 / Accepted 20 April 2016

\begin{abstract}
Aims. We study the different patterns of interannual magnetic variability in stars on or near the lower main sequence, approximately solar-type (G-K dwarf) stars in time series of $36 \mathrm{yr}$ from the Mount Wilson Observatory Ca II H\&K survey. Our main aim is to search for correlations between cycles, activity measures, and ages.

Methods. Time-frequency analysis has been used to discern and reveal patterns and morphology of stellar activity cycles, including multiple and changing cycles, in the datasets. Both the results from short-term Fourier transform and its refinement using the ChoiWilliams distribution, with better frequency resolution, are presented in this study. Rotational periods of the stars were derived using multifrequency Fourier analysis.

Results. We found at least one activity cycle on 28 of the 29 stars we studied. Twelve stars, with longer rotational periods $(39.7 \pm$ 6.0 days), have simple smooth cycles, and the remaining stars, with much faster rotation ( $18.1 \pm 12.2$ days) on average, show complex and sometimes vigorously changing multiple cycles. The cycles are longer and quite uniform in the first group $(9.7 \pm 1.9 \mathrm{yr})$, while they are generally shorter and vary more strongly in the second group (7.6 \pm 4.9$)$. The clear age division between stars with smooth and complex cycles follows the known separation between the older and younger stars at around 2 to 3 Gyr of age.
\end{abstract}

Key words. stars: activity - starspots - stars: late-type - stars: solar-type - Sun: activity

\section{Introduction}

Magnetically active stars including the Sun show multiple and variable cycles on different timescales with varying amplitudes (see Oláh et al. 2009, and references therein), thereby reinforcing the paradigm of change instead of constancy for solar and stellar activity outputs. In the Sun, cycles with different lengths, from the Rieger-type cycles (i.e., with a length or period shorter than $1 \mathrm{yr}$; see e.g., McIntosh et al. 2015), through cycles from $1 \mathrm{yr}$ to $22 \mathrm{yr}$ including the Schwabe sunspot cycle, to the Gleissberg and deVries-Suess cycles (i.e., timescales of several decades to two centuries) are often studied separately to explore their possible origins. With advances from studying the production of cosmogenic isotopes and the resulting indirect solar activity proxies in Earth archives, even cycles of millennial to bi-millennial timescales have been detected and researched (e.g., Soon et al. 2014). These cycles enrich models of dynamics of solar magnetic activity variations. The interrelations among these magnetic oscillations and characteristic timescales are a subject worthy of further investigation. The observational datasets for several decades that are available for stars that display magnetic activity in most cases are too short to derive long cycles (similar to the solar Gleissberg cycle) and barely enough to find cycles of several years, like the solar Schwabe cycle of 9 to $13 \mathrm{yr}$. That stars have multiple cycles has long been noted and discussed, but no particular attention was paid to them owing to limitations from both observational and theoretical points of view.

It is empirically known that the lengths of magnetic cycles are related to the stellar rotation rates: stars with slower rotation exhibit longer cycles, which was noted first by Baliunas et al. (1996). Surface magnetic activity and rotation are strongly related (cf. Reiners et al. 2014, and references therein), and as a star evolves, its rotation slows, changing the characteristics and operation of the presumed underlying dynamo as well. Therefore the pattern of long-term variation of active stars should reflect the working of the dynamo inside the stars. Such a relation has already been suggested as early as 1981 by Durney et al. (1981), based on results of the Mount Wilson Ca II H\&K survey (Wilson 1968), which had yielded time series for only about a decade at that time. The continued datasets from the Mount Wilson Observatory $\mathrm{Ca}$ II $\mathrm{H} \& \mathrm{~K}$ project survey, now three times as long, allow us to study and re-examine the suggestion of Durney et al. (1981) by using the observed patterns of the activity cycles in addition to adopting newly available basic parameters such as temperatures and ages of the stars. Our primary aim in this paper is to study whether the separation between young and old chromospherically active stars, first elaborated by Soon et al. (1993a) and later revisited by Brandenburg et al. (1998), can also be detected in the pattern or morphology of activity cycles.

The structure of our paper is as follows: in Sect. 2. we describe the data and the time-frequency methods, in Sect. 3. we 
present results of the time-frequency analysis, in Sect. 4. we discuss the results, and in Sect. 5. we summarize the study.

\section{Applied data and method}

We take advantage of the full 36 yr long Mount Wilson (MW afterwards) Ca II H\&K survey (Wilson 1968, 1978; Baliunas et al. 1995) dataset for 29 stars of spectral type G-K to deduce surface rotational modulation periods and activity cycles on an about $10 \mathrm{yr}$ longer time-base than was previously available. Wilson originally chose the sample as single (i.e., not close binaries, although several wide binary pairs were included) stars on or near the lower main sequence based on parameters known in the mid1960s. Modern refinements to those parameters for stars in the sample are discussed below.

Additionally, we studied the newest version of the sunspot number dataset published by WDC-SILSO, Royal Observatory of Belgium, Brussels. The sunspot data were binned to $0.025 \mathrm{yr}$ ( $\approx 9$ days). For comparison and verification purposes, we made use of the disk-integrated solar Ca II K-line flux measurements from the Evans Coronal Facility at The National Solar Observatory/Sacramento Peak observed between 1976-2015 ${ }^{1}$. The data were binned (and if necessary, interpolated) to 30 days.

To study activity cycles in the sample of 29 stars from the MW survey, we used the same time-frequency analysis as in Oláh et al. (2009). Briefly, we used a short-term Fourier transform (STFT) that gives acceptable resolution both in time and in frequency domains. The observational noise and the used spline interpolation do not alter the frequency distribution, as was discussed in detail by Kolláth \& Oláh (2009) using Monte Carlo simulations. We also ran the analysis using a Choi-Williams distribution (CWD), which has a higher frequency resolution, at the cost of the time resolution or cross terms, to verify the previous cycle length determinations. For the Sun, we applied different amplifications for the amplitudes in different frequency intervals for better visibility of the results. Details about the time-frequency analysis program are found in Kolláth \& Oláh (2009). We extracted rotational periods from the data with the multiple-frequency analysis tool MuFrAn (Kolláth 1990; Csubry \& Kolláth 1990). The shorter cycles on an interannual timescale are generally not always detectable in stellar activity data records owing to irregular and rather limited seasonal time sampling.

We first discuss shorter term cycles on the Sun which coexist with the Schwabe cycle simultaneously and may add insight on the physical manifestations of the solar dynamo operation and add confidence in studying and interpreting such shorter term activity oscillations should they also be found in stellar activity records of the MW H\&K survey.

\section{Results}

We define "dominant cycles" of the stars as the most evident cycles with a high amplitude, which are at the same time repeated at least twice during the observational time window. A high amplitude of the dominant cycle means that the cycle has the highest amplitude during the observed time interval, except for some cases when the longest term trends and tendencies of the data records yield an even higher amplitude. The MW datasets are too short (36 yr) to define longer cycles such as the solar Gleissberg and deVries-Suess cycles (i.e., 50-200 yr), but the longest term trends found in many stars could be parts of such long cycles. Many of the dominant cycles, however, are reminiscent of

1 http://lasp.colorado . edu/lisird/tss/cak.html the solar Schwabe (11 yr) cycle, which is generally referred to as "solar cycle" in this paper.

\subsection{Quasi-biennial oscillations of the Sun}

Shorter cycles of the Sun are well known. They range from Rieger-type cycles on timescales of shorter than 1 yr to the so-called mid-term cycles or quasi-biennial oscillations (QBO) with typical lengths of 1-4 yr. However, neither Rieger-type cycles nor mid-term cycles, which are studied in detail concerning both their time behavior and possible origin (cf., e.g., Forgács-Dajka \& Borkovits 2007), are continuously present in the solar datasets. Bazilevskaya et al. (2014) published an excellent review about the QBOs, and we refer to this paper and references therein for further information. McIntosh et al. (2015) also recently added details of and insights into these characteristic variations and modeled them as overlapping solar magnetic activity band interactions and instabilities.

We analyzed the most recent version of the sunspot number dataset between 1818-2015 (WDC-SILSO, Royal Observatory of Belgium, Brussels) with the same method as used for photometry and/or the Ca index data of stars (Oláh et al. 2009). The result is presented in Fig. 1. Varying cycles on four different timescales are shown in the left panel of Fig. 1. The longest of these is the Gleissberg cycle; the next three are the Schwabe (11 yr) cycle and its half and one-third harmonic components. Mixed with the one-third component of the fundamental Schwabe cycle, an independent cycle is found that is also variable, changing between 3 and $4 \mathrm{yr}$. We only mention this cycle and consider it a plausible upper-end limit of the QBO activity variations. However, it should be noted that Barnhart \& Eichinger (2011), using empirical mode decomposition (EMD) combined with the Hilbert transform, found 3.4-3.6 yr periods from sunspot numbers, although with a high uncertainty of about $50 \%$, as the shortest mode from their analysis. Our independent result shows that a cyclic variability on a timescale of 3-4 yr may be continuously present in solar variability and is manifested in the sunspot number. This is consistent with the recent interpretation by McIntosh et al. (2015), for example.

The solar Ca II K-line flux dataset is similar to the data of the MW stars both in length (only about three years longer) and measure. Figure 1 (middle and right panels) shows the resulting time-frequency analysis for a shorter time-interval for the sunspot number and the solar Ca II K-line flux. The similarity is evident; the cycle of 3-4 yr is also clearly detected in the Ca II Kline flux data and adds confidence to our interpretation.

\subsection{Cyclic changes on different timescales of MW stars}

We next analyzed Ca-index measurements from the Mount Wilson Observatory for 29 stars. The time series of MW stars have about the same length of $\approx 36 \mathrm{yr}$ and serves as a source in studying relations among stellar rotation, cycles, and other parameters. We found multiple cycles on the stars in most cases, of which several are also variable in time. Some of the cycles are only temporarily visible, while others are present during the entire observed time span, similarly to the solar case. We did not use any special treatment to obtain the cycle lengths, which should be considered more as characteristic timescales of certain physical mechanisms or processes and therefore are not strictly periodic. Most of these cycles and even their variabilities are clearly visible on the datasets themselves, and the numerical values were taken interactively from the time-frequency diagrams. 


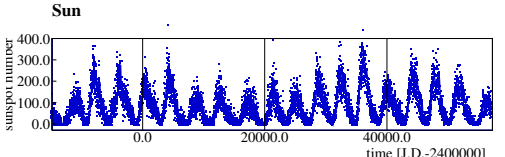

Time-frequency distribution (STFT):

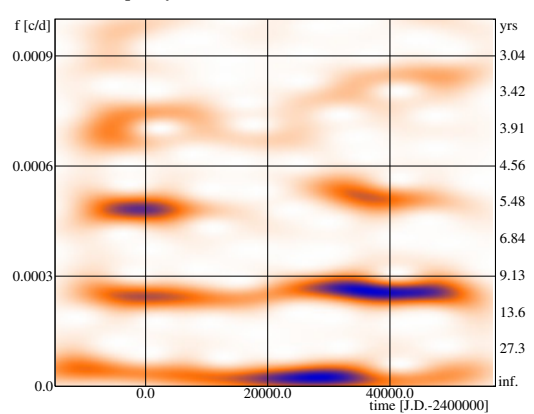

Sun

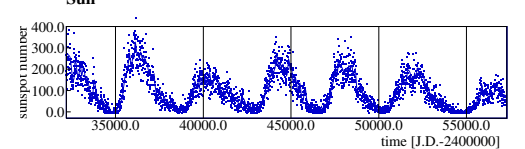

Time-frequency distribution (STFT):

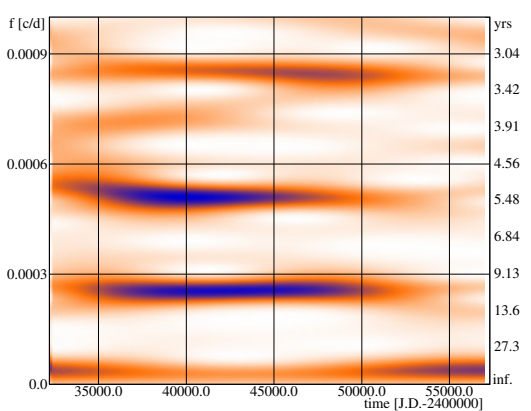

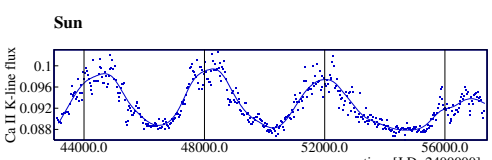

Time-frequency distribution (STFT):

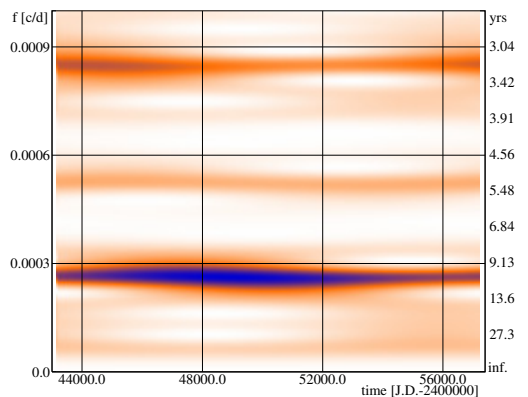

Fig. 1. Solar data (upper panels) and STFT calculations (lower panels). Left: sunspot number data from 1818 to the present and STFT. The Gleissberg cycle is shown in the bottom of the figure; above this is the Schwabe (11 yr) cycle, and above this, its half and one-third harmonic cycles are also found. Additionally, and mixed with the one-third of the Schwabe cycle, another changing variation of 3-4 yr is continuously present in the data. Middle: STFT of a shorter record of sunspot number (1947 to present) and right: the same for the Ca II K flux of the Sun between 1976-2015. The variability of 3-4 yr is also well detected in the two shorter datasets.

The type of the long-term variability of the MW stars divides the sample into two classes by the simple or complex nature of their dominant cycles. Twelve stars show a high-amplitude slow variability with clearly detected cycles, and sixteen have more erratic variations. The STFT maps are plotted in Figs. 2 and 3 for the two types, see also the Appendix for the confirmatory CWD maps. The numerical results are given separately for the two groups in Table 1. In the table we list spectral types from different sources (Gray et al. 2003, 2006; Baliunas et al. 1995), rotational periods, and mean cycle lengths determined in this paper. For changing cycles, the extreme values or limits of the cycle lengths are also listed in parentheses. When available, cycle lengths from Baliunas et al. (1995) are given for comparison in the last column. Only one star (HD 165341B) does not have any cycle; its time series displays only a slow long-term variation in the course of the observations. Altogether, 14 stars show more than one cycle, and 27 stars (except HD 16160) have at least one changing cycle.

When we compare the results of the derived cycles with those published by Baliunas et al. (1995), we find a very good general agreement. Baliunas et al. (1995) used Lomb-Scargle periodograms for the datasets, which were about ten years shorter than in the present paper. Their published errors of the cycle lengths could reflect changing cycles: for HD 100180, for instance, we find two cycles of $3.63 \mathrm{yr}$ and a variable cycle of between 16.6-9.85 yr, while Baliunas et al. (1995) derived $3.56 \pm 0.04$ and $12.9 \pm 0.5 \mathrm{yr}$. Our newly determined rotational periods from the longer dataset are within the minimum and maximum periods from the seasonal results of Donahue et al. (1996) for the stars in common with the present MW sample. For $17 \mathrm{MW}$ stars, we find long-term changes that might be parts of other variability on long timescales, similar to the solar Gleissberg cycle, for example.

Table 1 clearly shows that the 12 stars with simple cycle patterns have generally longer rotational periods of $39.7 \pm 6.0$ days (median: $39.3 \mathrm{~d}$ ) and corresponding cycle lengths of $9.7 \pm 1.9 \mathrm{yr}$ (median: $9.5 \mathrm{yr}$ ), respectively. Some of those stars have shorter secondary cycles with low amplitudes of a few percent of the main cycle amplitude on timescales of around 3.5-4 yr. The rotation and cycle lengths show a much higher dispersion for 16 stars with complex cycles: their rotation of $18.1 \pm 12.2$ days (median: $14.9 \mathrm{~d}$ ) is on average half as long as that of the other group, and the scatter is on the same order as the value itself. The average cycle length is $7.6 \pm 4.9 \mathrm{yr}$ (median: $5.2 \mathrm{yr}$ ), meaning that it is also shorter and more scattered than for the stars with simple cycles. Moreover, the stars with complex cycle variations show secondary and sometimes tertiary cycles that can be shorter or longer than the dominant cycle, with amplitudes ranging between $5-65 \%$ of the dominant cycles. Figures 2 and 3 show the striking difference between the long-term changes of the two groups.

The recent detection of dual magnetic activity cycles at $1.7 \mathrm{yr}$ and about $12 \mathrm{yr}$ in the rapidly rotating solar analog star HD 30495 fit our results well. It has a spectral type G $1.5 \mathrm{~V}$ $\left(P_{\text {rot }}=11\right.$ days $)$ and an estimated age of about 1 Gyr as reported by Egeland et al. (2015). Furthermore, because of the longer observational records available, those authors were also able to discern that the individual decades-long cycles have durations ranging from 9.6 to $15.5 \mathrm{yr}$. In addition, Metcalfe et al. (2013) also reported the detection of simultaneous operation of two stellar activity cycles of $2.95 \pm 0.03 \mathrm{yr}$ and $12.7 \pm 0.3 \mathrm{yr}$ for the young star HD 22049 ( $\epsilon$ Eridani), which hosts a planet with absolute mass of 15 times the mass of Jupiter. Findings of multiperiodicity by other authors are consistent with similar results presented in our Figs. 2 and 3.

\section{Discussion}

The rotational and cycle periods are two important observables of magnetically active stars. Their ratio, $P_{\text {cyc }} / P_{\text {rot }}$ can be related to the dynamo number $N_{D}$, giving a directly measurable parameter that is closely related to the magnetic activity of the stars. It was first proposed by Soon et al. (1993a). For a detailed discussion of this topic, see Baliunas et al. (1996), who, based on the observations of MW stars with a shorter time-base $(\approx 25 \mathrm{yr})$ at that time, stressed the importance of the measured quantities in examining stellar dynamos.

Durney et al. (1981), and more recently Böhm-Vitense (2007), studied the rotations and cycles of MW stars as a function of their temperatures. We re-examine the relation for the stars of our sample plotted in Fig. 4. Effective temperatures, when available, are taken from Gray et al. (2003, 2006). For stars 

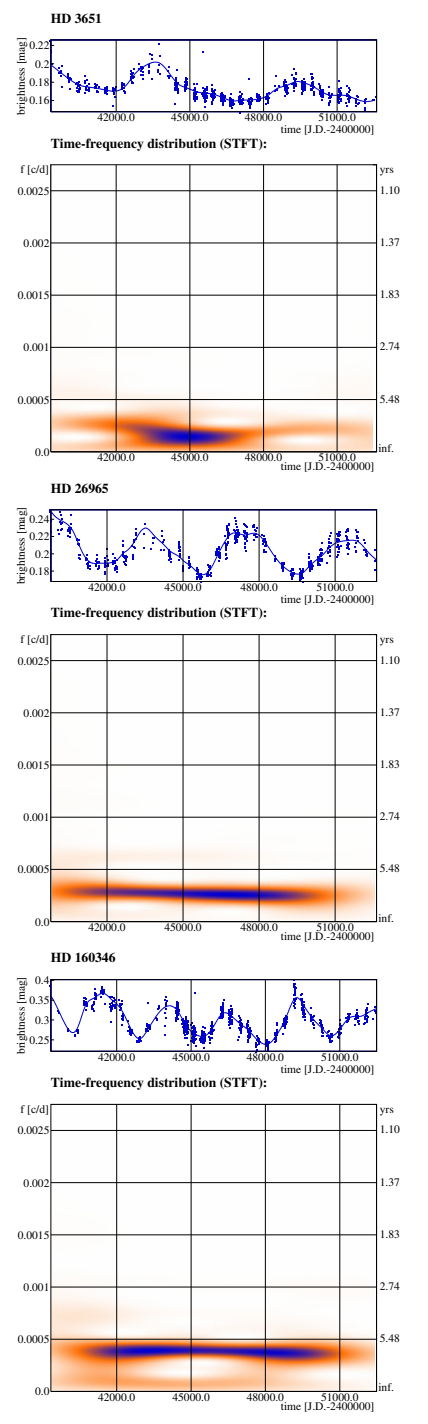

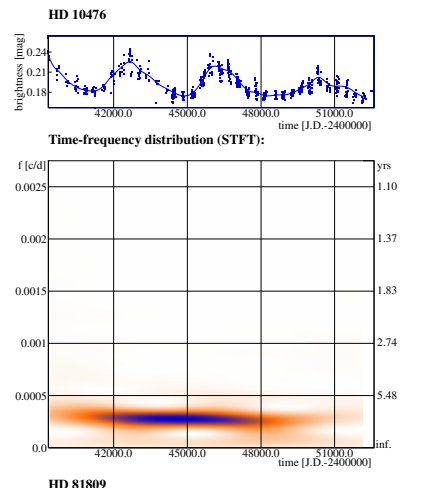

HD 32147
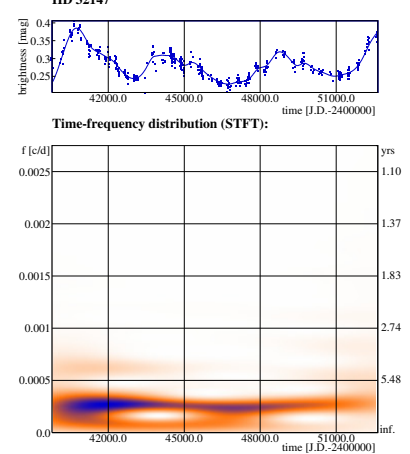

HD 166620
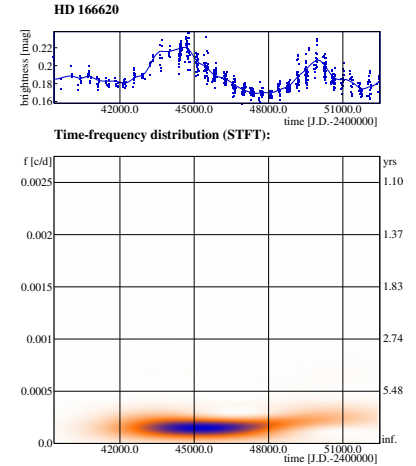
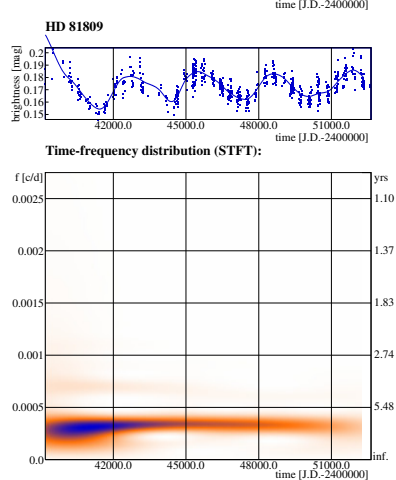

HD 201091
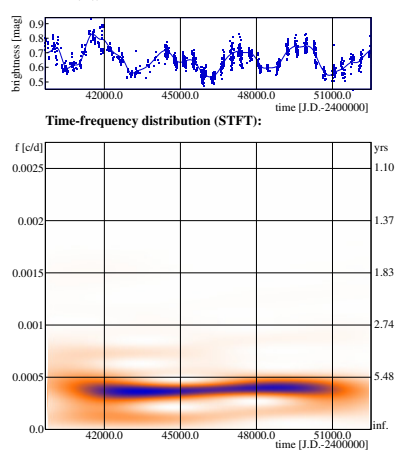

HD 16160

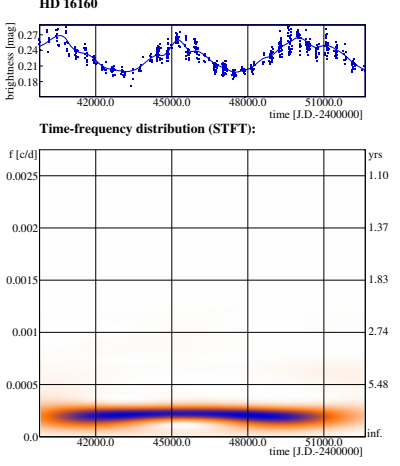

HD 103095
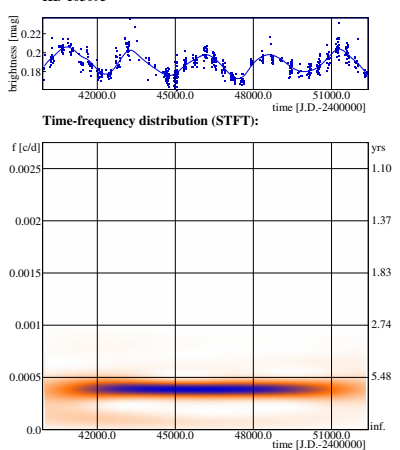

HD 219834B
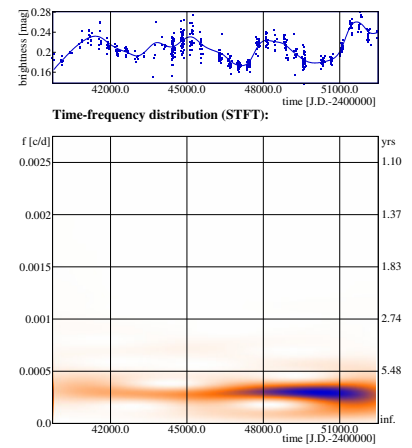

Fig. 2. STFT of MW stars with simple cycles. Upper panels: datasets and spline interpolation; lower panels: time-frequency diagrams. We applied no amplification, therefore the strengths of the low-amplitude secondary cycles can be directly compared. (However, in a few cases the low-amplitude signals are too faint in the prints.)

without temperatures in those two papers, we took spectral types from Baliunas et al. (1995) and used the color-temperature calibration of Pecaut \& Mamajek (2013) to calculate effective stellar temperatures. From comparing the temperatures of the 18 stars from Gray et al. (2003, 2006) with the color-temperature calibration of the same stars from Pecaut \& Mamajek (2013), we find on average temperature differences smaller than $60 \mathrm{~K}$, with the latter calibration being hotter. However, the small difference in temperature calibration methods does not affect our result, which is depicted in Fig. 4.

The rotational periods show a bimodal distribution between the stars with simple and complex cycles as a function of effective temperature, as shown in the top panel of Fig. 4. The picture is the same as in Böhm-Vitense (2007), her Fig. 4, showing the difference between the active (A) and inactive (I) stars; the different dynamos working in the stars of the two groups were given as the possible reason of the bimodality in that paper. In the bottom panel of Fig. 4, no strong correlation is seen for the stars with simple cycles, and a possible slight increase of $P_{\text {cyc }} / P_{\text {rot }}$ is found for the stars with complex cycles toward higher temperatures, that is, for earlier type stars. We note that the coolest star, HD 95735, which is an M-dwarf, is excluded from the two relations of Fig. 4 because its internal structure may differ significantly from the other stars. The MW survey was able to detect this convective M-dwarf while it was flaring in real time during the 1981 observing season; the data points are plotted in Fig. $1 \mathrm{~g}$ (right panel, bottom) of Baliunas et al. (1995).

We plot $P_{\text {cyc }} / P_{\text {rot }}$ as a function of $1 / P_{\text {rot }}$ in Fig. 5 similar to the figure first shown by Baliunas et al. (1996), displaying all cycles derived in this paper and given in Oláh et al. (2009) using the same time-frequency analysis method. In addition to the MW stars, the plot contains fast-rotating stars, both singles and binaries, and also evolved stars. The fit, with a slope of $0.76 \pm 0.15$, was made only for the dominant periods of MW stars derived in this paper (large symbols), based on the Caindex measurements, that is, essentially the same as in Baliunas et al. (1996). The relation matches the region of the fast-rotating K-dwarf stars ( $P_{\text {rot }}$ is between about 1.5-3 days) and does not contradict the results for evolved stars; we note that all results from Oláh et al. (2009) are plotted with small green dots. However, the samples of the cycles derived from photometry (i.e., photospheric variability) for fast-rotating stars of different types, that is, single stars, binaries, dwarfs, and giants, are too small to see if there is any basic difference between the cycle patterns of 
K. Oláh et al.: Magnetic cycles at different ages of stars
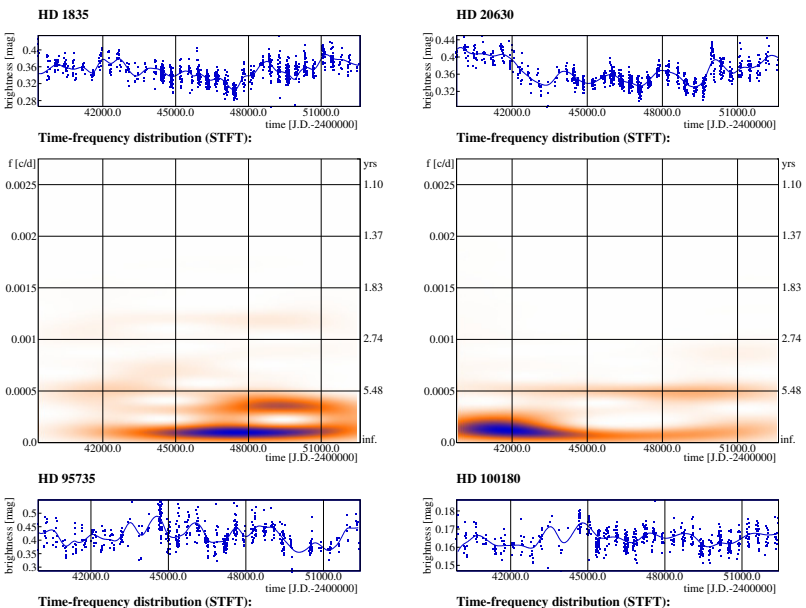

HD 10018

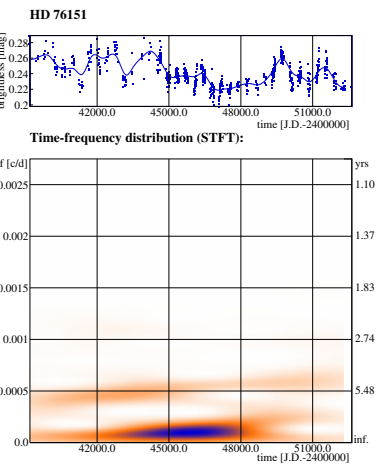

HD 78366

Time-freepuency distribution (STFT):

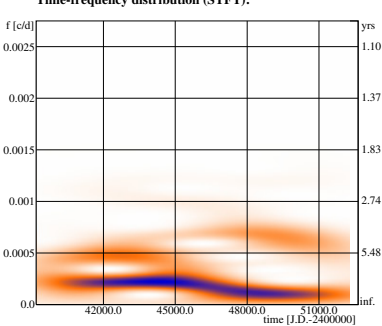

114710

HD 115404

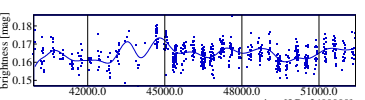

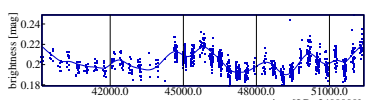

Time-frequency distribution (STFT)

Time-frequency distribution (STFT):
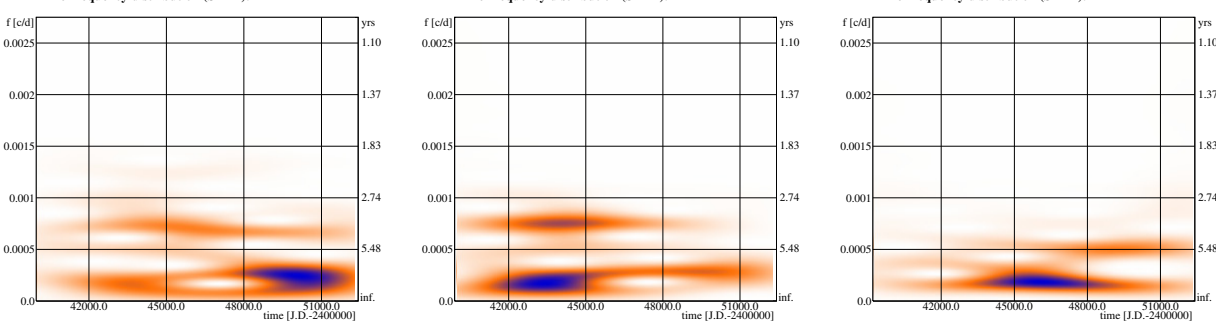

HD 131156B

HD 149661

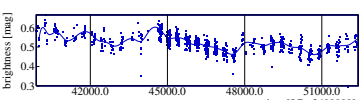

Time-frequency distribution (STFT):

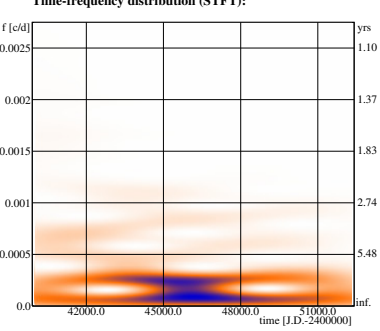

HD 152391

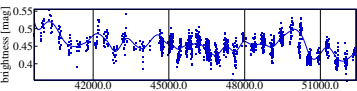
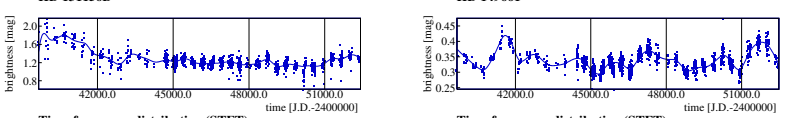

Time-frequency distribution (STFT):

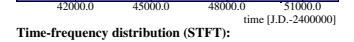

Time-frequenters
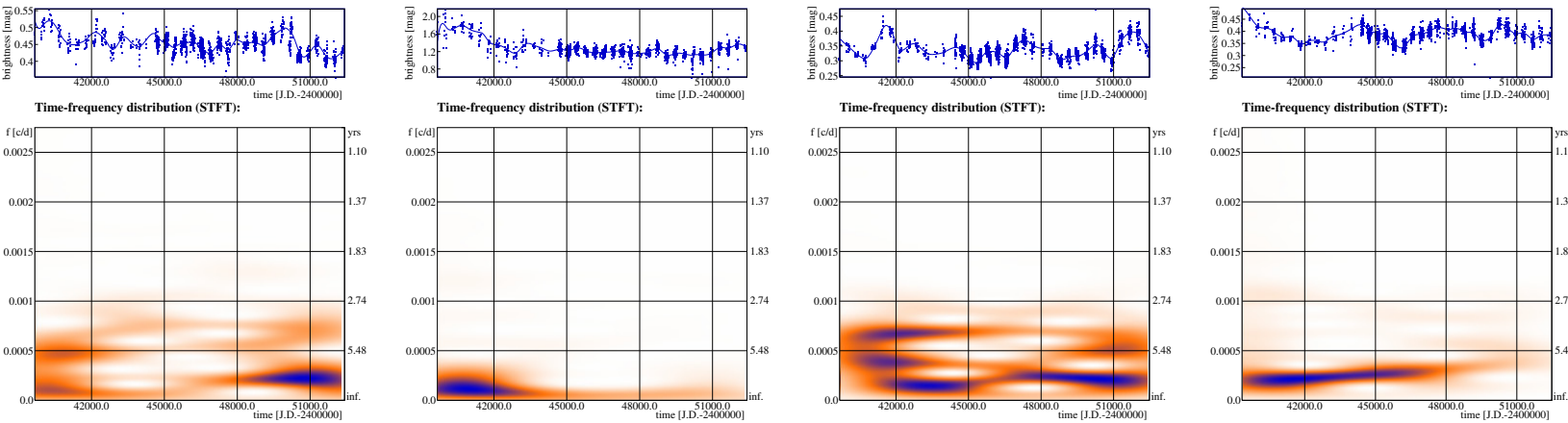

HD 156026

HD 165341A

HD $165341 \mathrm{~B}$

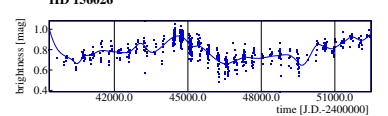

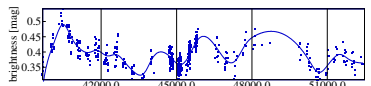

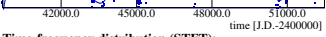

Time-frequency distribution (STFT):
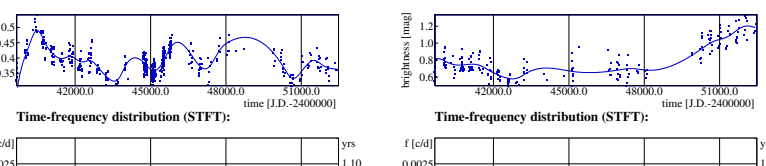

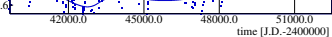

Time-frequency distribution (STET):
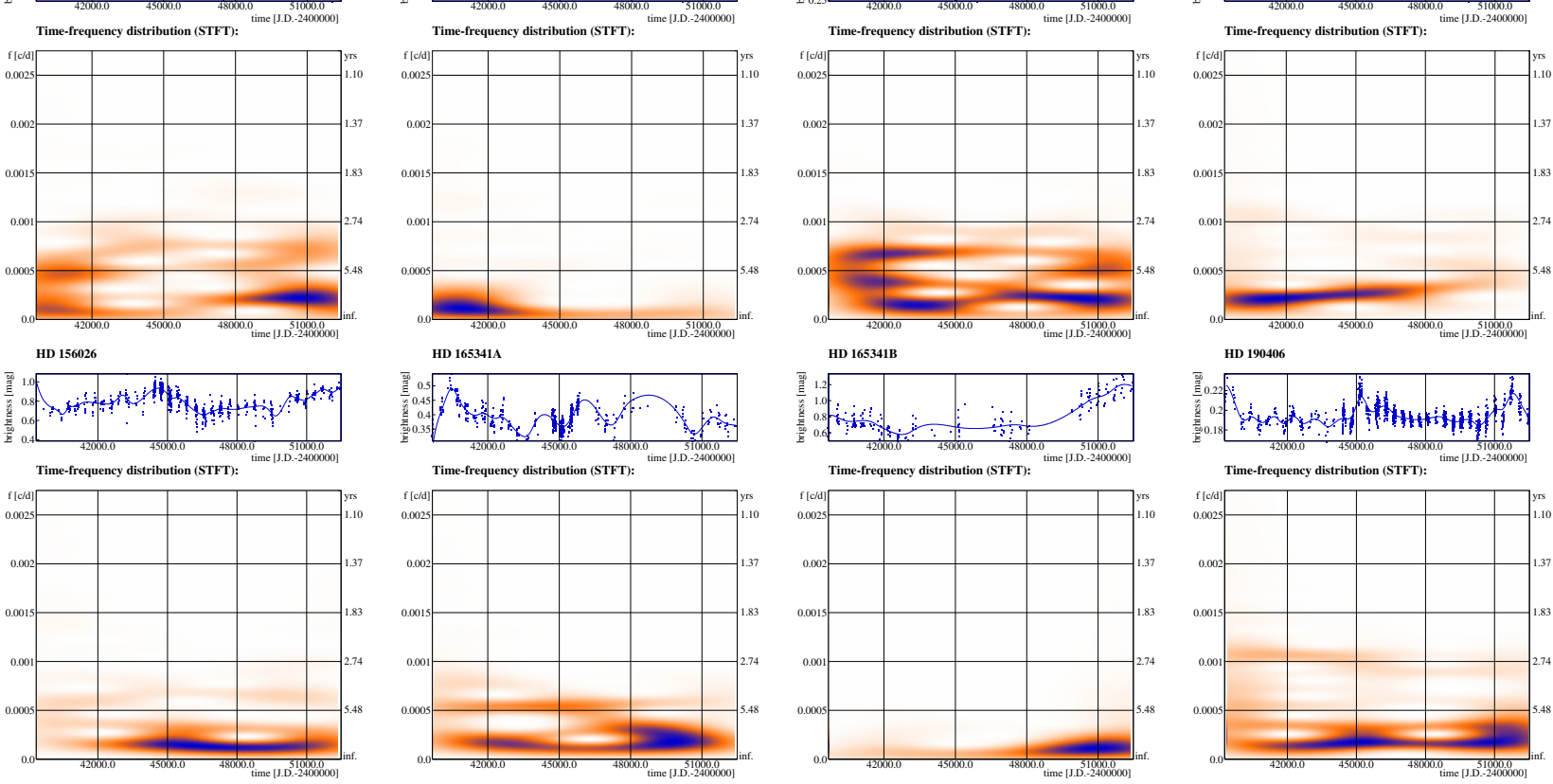

HD 190406

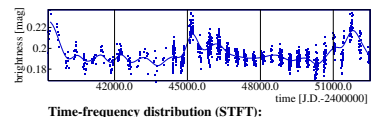

Time-frequency distribution (STFT):

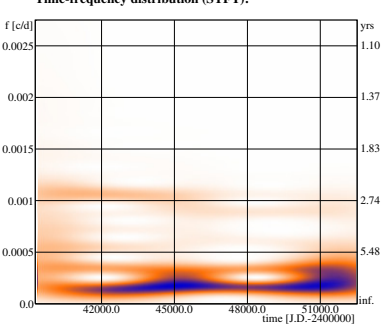

HD 201092

42000.0

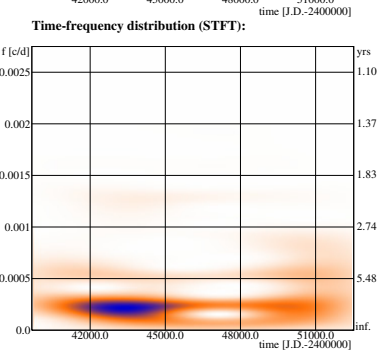

Fig. 3. STFT of MW stars with complex cycles. Upper panels: datasets and the spline interpolation; lower panels: time-frequency diagrams. We applied no amplification, therefore the cycle amplitudes can be directly compared. 
Table 1. All detected cycles in the MW stars; the first is the dominant cycle.

\begin{tabular}{|c|c|c|c|c|c|}
\hline Star & Sp. type & $\begin{array}{l}P_{\text {rot }} \\
\text { (days) }\end{array}$ & $\begin{array}{l}P_{\text {cyc,(range) }} \\
\text { (years) }\end{array}$ & $1 t^{c}$ & $\begin{array}{l}P_{\text {cyc }}, \text { Baliunas et al. (1995) } \\
\text { (years) }\end{array}$ \\
\hline \multicolumn{6}{|c|}{ simple cycles } \\
\hline HD 3651 & $\mathrm{~K}_{0} \mathrm{~V}^{a}$ & $44^{b}$ & $11.6(9.57-13.7)-\mathrm{lt}$ & yes & $13.8 \pm 0.4$ \\
\hline HD 4628 & $\mathrm{~K} 2.5 \mathrm{~V}^{a}$ & 41.6 & $8.94(8.30-9.5)$ & no & $8.37 \pm 0.08$ \\
\hline HD 10476 & $\mathrm{~K} 1 \mathrm{~V}^{a}$ & 33.7 & $9.8(9.01-9.85)$ & no & $9.6 \pm 0.1$ \\
\hline HD 16160 & $\mathrm{~K} 3 \mathrm{~V}^{a}$ & 57 & 12.1 & no & $13.2 \pm 0.2$ \\
\hline HD 26965 & $\mathrm{~K} 1 \mathrm{~V}^{a}$ & $43^{b}$ & $10.0(9.57-10.5)$ & no & $10.1 \pm 0.1$ \\
\hline HD 32147 & $\mathrm{~K} 3+\mathrm{V}^{a}$ & 39.3 & $10.6(9.85-11.3), 1 \mathrm{t}$ & yes & $11.1 \pm 0.2$ \\
\hline HD 81809 & $\mathrm{G} \mathrm{V}^{a}$ & 39.3 & $8.69(8.10-9.28)$ & no & $8.17 \pm 0.08$ \\
\hline HD 103095 & $\mathrm{~K} 1 \mathrm{~V}^{a}$ & 36.5 & $6.95(6.9-7.0)$, lt & yes & $7.30 \pm 0.08$ \\
\hline HD 160346 & $\mathrm{~K} 2.5 \mathrm{~V}^{a}$ & 35.3 & $7.35(7.2-7.5), \mathrm{lt}$ & yes & $7.00 \pm 0.08$ \\
\hline HD 166620 & $\mathrm{~K} 2 \mathrm{~V}^{c}$ & 41.5 & $13.6(9.6-17.6)$ & no & $15.8 \pm 0.3$ \\
\hline HD 201091 & $\mathrm{~K} 5 \mathrm{~V}$ & 37.1 & $6.95(6.7-7.2), \mathrm{lt}$ & yes & $7.3 \pm 0.1$ \\
\hline HD 219834B & $\mathrm{K} 2 \mathrm{~V}$ & 34.0 & $9.29(9.01-9.57)$ & no & $10.0 \pm 0.2$ \\
\hline Sun & $\mathrm{G} 2 \mathrm{~V}$ & 27.275 & $11(9-14), 3.65(3.3-4.0)^{d}$ & yes & \\
\hline \multicolumn{6}{|c|}{ complex cycles } \\
\hline HD 1835 & $\mathrm{G} 2 \mathrm{~V}^{a}$ & 7.84 & 7.6(7.3-7.9), 2.4(2.50-2.28), 3.97(4.85-3.09), lt & yes & $9.1 \pm 0.3$ \\
\hline HD 20630 & G5V & 9.08 & $5.32(5.36-5.27)$ & no & $5.6 \pm 0.1$ \\
\hline HD 76151 & $\mathrm{G} 3 \mathrm{~V}^{a}$ & 15.2 & $5.32(6.07-4.56)$, lt & yes & $2.52 \pm 0.02$ \\
\hline HD 78366 & G0V & 9.7 & $13.45(12.6-14.3)-1 t, 4.0(3.85-4.15)$ & yes & $12.2 \pm 0.4,5.9 \pm 0.1$ \\
\hline HD 95735 & M2V & 54 & $3.90(3.75-4.04), 12.7(10.3-15.0)-1 \mathrm{t}$ & yes & \\
\hline HD 100180 & $\mathrm{~F} 9.5 \mathrm{~V}^{a}$ & 14.6 & $13.2(16.6-9.85), 3.63$ & no & $3.56 \pm 0.04,12.9 \pm 0.5$ \\
\hline HD 114710 & G0V & 12.9 & 16.6(17.6-13.7-18.6), 7.7(7.2-8.2), 5.25(5.1-5.4) & no & $16.6 \pm 0.6,9.6 \pm 0.3$ \\
\hline HD 115404 & $\mathrm{~K} 2.5 \mathrm{~V}^{a}$ & 18.8 & $10.8(9.57-12.1), 5.08(5.83-4.33), 3.4(3.96-2.84) \mathrm{lt}$ & yes & $12.4 \pm 0.4$ \\
\hline HD 131156A & G8V & 6.25 & $3.7(3.6-3.8), 12,1 \mathrm{t}$ & yes & \\
\hline HD $131156 \mathrm{~B}$ & $\mathrm{~K} 4 \mathrm{~V}$ & 11.05 & $4.2(4.64-3.76), 2.28, \mathrm{lt}$ & yes & \\
\hline HD 149661 & $\mathrm{~K}_{0} \mathrm{~V}^{a}$ & 21.3 & $4.0,6.2(5.6-6.8), 12.25(11.3-13.2)-\mathrm{lt}$ & yes & $17.4 \pm 0.7,4.00 \pm 0.04$ \\
\hline HD 152391 & $\mathrm{G} 8+\mathrm{V}^{a}$ & 11.4 & $9.9(12.6-7.17), 2.8(2.5-3.1)$ & no & $10.9 \pm 0.2$ \\
\hline HD 156026 & $\mathrm{~K} 5 \mathrm{~V}$ & 29.2 & $4.33(3.95-4.71), 8.1, \operatorname{lt}(\approx 20)$ & yes & $21.0 \pm 0.9$ \\
\hline HD 165341A & $\mathrm{K}_{0} \mathrm{~V}^{a}$ & 18.9 & $11.4(9-13.8), 5.17, \mathrm{lt}$ & yes & $5.1 \pm 0.1$ \\
\hline HD $165341 B$ & K6V & - & - & & Var \\
\hline HD 190406 & $\mathrm{G} \mathrm{V}^{a}$ & 15.5 & 15.8(15.0-17.6)-lt, 8.35(7.69-9.01), 4.65(4.44-4.86), 2.33, 3.01 & yes & $2.60 \pm 0.02,16.9 \pm 0.8$ \\
\hline HD 201092 & K7V & 34.1 & $13.4(10.1-16.6)-1 t, 4.45(4.71-4.18),(2.07:)$ & yes & $11.7 \pm 0.4$ \\
\hline
\end{tabular}

Notes. In the last column we list results from Baliunas et al. (1995) for comparison. ${ }^{(a)}$ Spectral types are taken from Gray et al. (2003, 2006), otherwise from Baliunas et al. (1995); ${ }^{(b)}$ period form Barnes et al. (2007); ${ }^{(c)}$ lt: long-term trend in the data; ${ }^{(d)}$ determined in the present paper.

these active stars that concerns their binarity and/or evolutionary status. The general trend between the rotation and cycle length plotted in Fig. 5 looks quite similar for all types of active stars, also regardless of the source of the data (chromosphere or photosphere). Values arising from the secondary and tertiary cycles of the MW stars fall within the scatter of the relation.

Several attempts have been made to connect rotation, activity cycles, and ages with the observed properties of the MW stars. The closest study to ours is that of Soon et al. (1993a), their Fig. 2, which shows a clear separation in $\log \left(P_{\text {cyc }} / P_{\text {rot }}\right)^{2}$ between older and younger stars and is interpreted as a consequence of the change of the dynamo efficiency as stars evolve. To construct a similar diagram, we used the pattern of the long-term variability of the stars (simple-complex cycles and cycle lengths) and their estimated gyro ages from Barnes (2007). The errors of the gyro ages are between 13-20\% and are not plotted for clarity. In his Table 3, Barnes (2007) lists the ages of the MW stars of our sample derived from isochrone fitting, activity-rotation relation, and his own technique. The $25 \%$ discrepancy between the chromospheric and gyro ages of the MW stars arises in stars with $B-V<0.6$, that is, from the bluer stars earlier than $\mathrm{G} 0-1 \mathrm{~V}$ with $T_{\text {eff }}$ hotter than about $6000 \mathrm{~K}$. We consider this discrepancy to be not so serious as to render the determined age to be incorrect.

We find a clear separation between the stars with simple and complex cycle patterns as a function of age in the sense that stars with simple cycles are older, as seen in the top panel of Fig. 6. The separation occurs near the age of the Vaughan-Preston gap (i.e., a bifurcation in the activity owing to the lack of stars with intermediate activity, Vaughan \& Preston 1980) at about 2.2 Gyr (Donahue 1996) and indicates a change in the dynamo. (The M-dwarf HD 95735, slightly older than 3 Gyr, is not part of the relation and is not plotted.) The morphology of stellar activity cycles appears to undergo a characteristic switch at a transitional age and clearly requires a theoretical explanation.

The middle panel of Fig. 6 shows chromospheric emission as a fraction of luminosity, the dimensionless quantity $R_{H K}^{\prime}$ (Noyes et al. 1984) of the stars given in Barnes (2007) as a function of gyro age. The youngest and fastest rotating stars, younger than $\approx 1.5 \mathrm{Gyr}$, show much higher time-averaged activity than the rest of the sample. After this, a slow downward trend of the chromospheric activity with age is suggested by the data. 


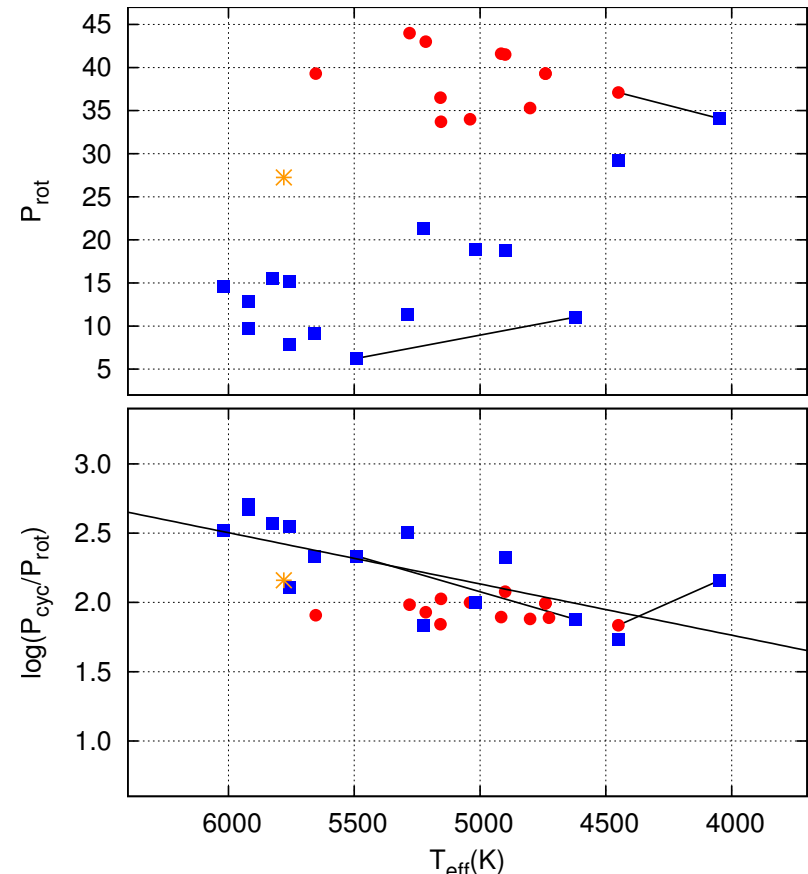

Fig. 4. Relations of rotational and cycle periods vs. $T_{\text {eff }}$. Stars with simple cycles are plotted with red dots, stars with complex cycles with blue squares, and the Sun with an orange star. The components of the wide binaries HD 131156A/B and HD 201091/HD 201092 are connected. Top: rotational periods versus effective temperatures. Bottom: $P_{\text {cyc }} / P_{\text {rot }}$ (related to the dynamo number) versus effective temperature. The fit for the stars with complex cycles shows a relation increasing toward hotter temperatures.

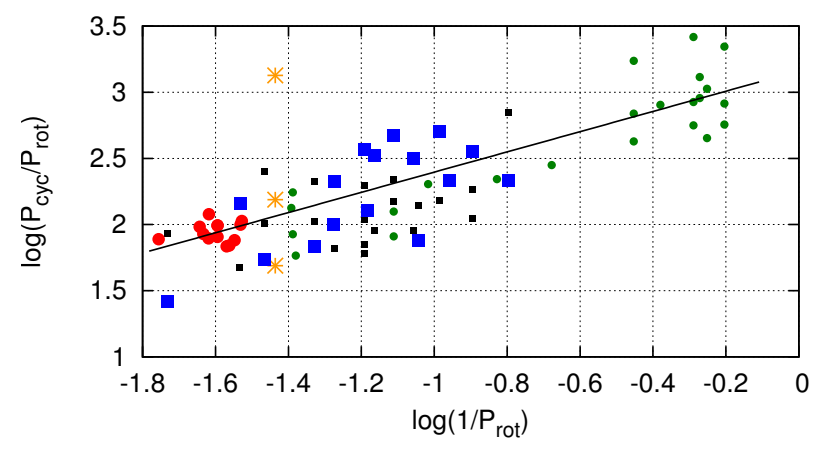

Fig. 5. Relation between the observed rotational and cycle periods. Stars with simple cycles are plotted with red dots, stars with complex cycles with blue squares, and the Sun with orange stars (Gleissberg, Schwabe, and 3-4 yr cycles). Small black squares denote additional cycles, and small green dots are the results from Oláh et al. (2009). The fit is only done for the dominant cycles derived in this paper. It traverses the region of the fast-rotating K-dwarf stars (singles and binaries) in the sample of Oláh et al. (2009). See the text for further discussion.

At first sight, the spread of data in the $P_{\text {rot }}$ and gyro ages in Fig. 6 seems too large to be physically meaningful. But we consider the possibility and reality of radial migration of individual stars, including the Sun and stars in the contemporary solar neighborhood, or even a whole cluster within the age of the Milky Way under the near-resonance interaction between the corotating star's angular momentum with the transient spiral density wave, which was recently studied by Roskar et al. (2012), for example. The spread of metallicity that may result from the migration of the stars in the solar neighborhood is more than 1.0 dex, without any reliable trend until at least 6-7 Gyr, as

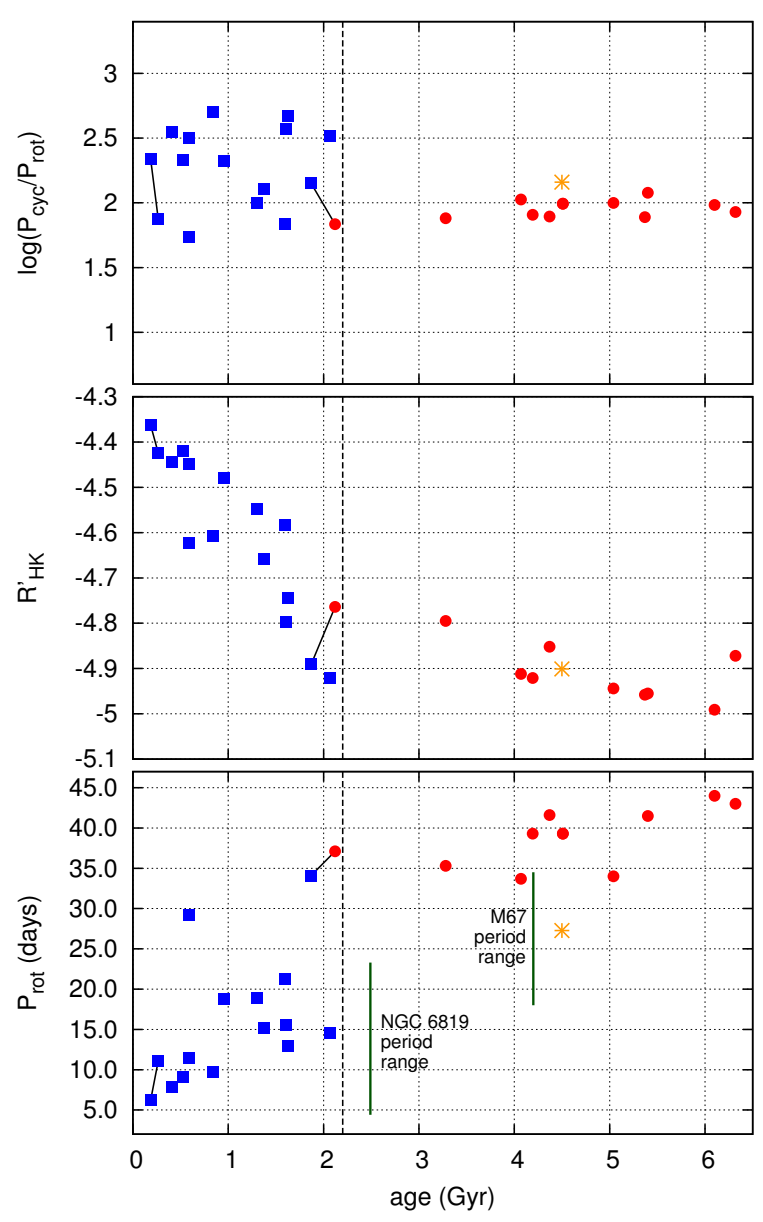

Fig. 6. Relations between the observed rotational and cycle periods, and $R_{H K}^{\prime}$ versus age. Stars with simple cycles are plotted with red dots, stars with complex cycles with blue squares, and the Sun with an orange star. The components of the wide binaries HD 131156A/B and HD 201091/HD 201092 are connected. Top: $P_{\text {cyc }} / P_{\text {rot }}$ show a clear separation between stars with simple and complex cycles in the sense that the simple cycles have about the same value, while complex cycles are more scattered. The division occurs near the age of the Vaughan-Preston gap (Donahue 1996). Middle: average $R_{H K}^{\prime}$ of the stars from Barnes (2007); the younger stars, especially below $1.5 \mathrm{Gyr}$, have a higher average activity. Bottom: $P_{\text {rot }}$ of the stars as a function of age, showing a clear separation, also near the Vaughan-Preston gap. The range of the rotational periods in NGC 6819 and M67 at their ages of $2.5 \mathrm{Gyr}$ and 4.2 Gyr are shown by green lines; data taken from Meibom et al. (2015) and Barnes et al. (2016).

plotted by Sellwood \& Binney (2002) in their Fig. 15. This picture is similar to ours for MW stars with available $\mathrm{Fe} / \mathrm{H}$ values (Gray et al. 2003, 2006) and based on the metallicity indicator $\left\langle C_{R V}\right\rangle$ (Soon et al. 1993b), which we plot in Fig. A.3. Such a large metallicity difference could indicate different evolutionary paths, with the full inclusion of the likely scenarios of radial migration within the Galaxy, even for stars of very similar mass as our MW sample, thereby accounting for at least some of the spread in Fig. 6.

A plausible explanation for the apparent dichotomy between stars of higher and lower level of activity (active vs. inactive stars) was suggested by Durney et al. (1981) as an explanation for the Vaughan-Preston gap. In their scenario, several dynamo modes are excited in stars with a rotation rate higher than a critical value, leading to a higher overall level of activity. In slower rotating stars, in contrast, only the fundamental mode 
is excited. Our results seem to agree with this general scenario: a difference in the overall activity level $\left(R_{H K}^{\prime}\right)$ across the V-P gap is quite obvious from our data. In addition, the bottom panel in Fig. 6 shows an indication of a non-uniform evolution of the rotation period.

Another possibility is that the nonlinear dynamo switches from one saturated state to another. This possibility arises in the bimodal interface dynamo proposed by Petrovay (2007). In this dynamo, two alternative solutions exist under fairly general assumptions on the nonlinearity (some of which were confirmed by Petrovay et al. 2010): a strong-field, short-period solution with a thin tachocline, and a weak-field, long-period solution with a thicker tachocline. A transition from the latter to the former state is expected to lead to a shortening of the dominant dynamo period and to an increase in the level of activity compared to what is expected from the extension of the active branch toward a lower rotation rate. Some support for the bimodal interface dynamo may be present, as shown in the middle panel of Fig. 6. (In this sense, using the term "inactive branch" for the regular group may be misleading.) In addition, the reorganization of the tachocline may potentially lead to a change in the overall rotation rate of the convective envelope through an altered torque balance. On the other hand, this scenario in its present form offers no explanation of the complex multiperiodic variation seen in the presumed weak-field dynamo.

\subsection{Validity of gyro ages for the MW stars}

Meibom et al. (2015) selected 30 stars in NGC 6819, an open cluster of about $2.5 \mathrm{Gyr}$, to study their rotation with high precision. They found a clear relation between the rotational periods and stellar masses at the age of the cluster, which supports the validity of the rotational evolution model of Barnes (2010) with the best fit of the rotational period versus $(B-V)_{0}$ color index. More recently, Barnes et al. (2016) derived rotational periods of 20 cool stars in the 4.2 Gyr old open cluster M67 based on data from the Kepler $\mathrm{K} 2$ mission and also found a good relation between the rotational periods and $(B-V)_{0}$ color indices for stars with masses between $0.8-1.15 M_{\odot}$.

The bottom panel of Fig. 6 shows the rotational periods of MW stars as a function of age. The Vaughan-Preston gap is present, and the range of rotation periods is shown for the 30 stars in NGC 6819 by Meibom et al. (2015) at the cluster age of $2.5 \mathrm{Gyr}$ and for the 20 stars in M67 by Barnes et al. (2016) at the cluster age of 4.2 Gyr. The period range of the NGC 6819 stars fits the range of the younger MW stars in the field well. The M67 results of Barnes et al. (2016) show that the longest period stars of this sample have $(B-V)_{0}$ of about 1.0 , corresponding to about $0.8 M_{\odot}$ and matching our results for the older MW stars with similar masses (see below), near the age of the Sun. The gyro ages of the MW stars and that of NGC 6819 and M67 clusters were derived based on the same background, using the expressions of Barnes $(2007,2010)$.

Almost all stars of our sample (cf. Fig. 4) fall into the temperature region where chromospheric and gyro ages agree well with each other. van Saders et al. (2016) found anomalously rapid rotation among old field stars based on rotational periods from Kepler data and astroseismically derived ages, which generally do not agree with the position of the older group of MW stars. In the top panel of Fig. 7, data from van Saders et al. (2016) are plotted with magenta crosses over our data. Four stars with $\log g>4.4$ from van Saders et al. (2016), marked with green circles in Fig. 7, fall near the positions of MW stars in both panels, showing that the relations given by the MW stars are valid

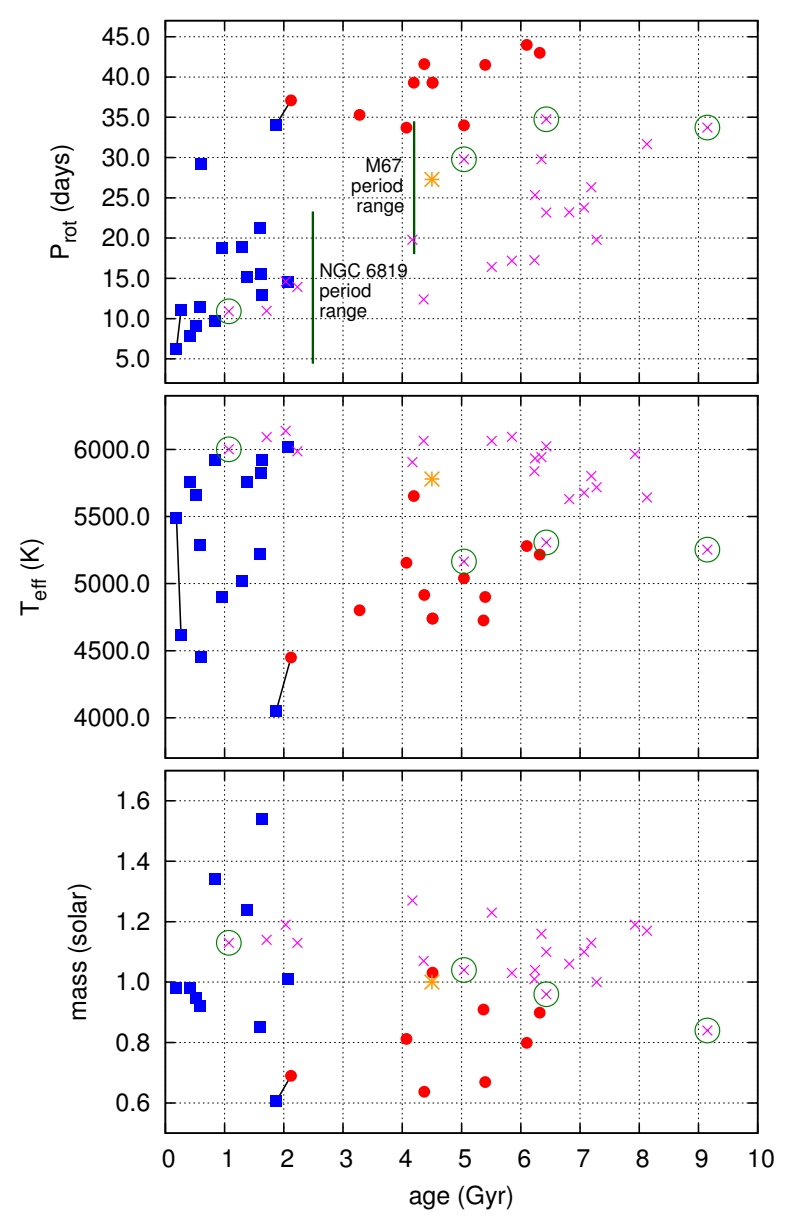

Fig. 7. Rotational periods and effective temperatures of the stars as a function of age. Stars with simple cycles are plotted with red dots, stars with complex cycles with blue squares, and the Sun with an orange star. Stars from van Saders et al. (2016) are plotted with magenta crosses, four stars with $\log g>4.4$ are marked with green circles. The components of the wide binaries HD 131156A/B and HD 201091/HD 201092 are connected. Top: rotational periods. The range of the rotational periods in NGC 6819 and M67 at their ages of $2.5 \mathrm{Gyr}$ and $4.2 \mathrm{Gyr}$ are shown by green lines; data are taken from Meibom et al. (2015) and Barnes et al. (2016). Middle and bottom: effective temperatures and masses as a function of age, respectively. See the text for further discussion.

in a narrow $\log g$ range around 4.5 . We note that the average $\log g=4.50 \pm 0.17$ for $23 \mathrm{MW}$ stars with available values. In this average, we did not take HD 81809 into account $(\log g=3.84$, Gray et al. 2006) because it is a spectroscopic binary with components of 1.7/1.0 solar masses. Favata et al. (2008) and Barnes (2007) also noted that the gyro age of this object (10.6 Gyr) is uncertain because of its binarity; therefore, we used the chromoage $(4.19 \mathrm{Gyr})$ instead in the plots.

The middle panel of Fig. 7 shows the temperature vs. age for the same stars as in the top panel. Older than $4 \mathrm{Gyr}$, the stars from van Saders et al. (2016) are about $1000 \mathrm{~K}$ hotter than the MW stars of similar ages. The masses of the older MW stars are on average $0.79 \pm 0.13 M_{\odot}$ (masses are taken from Valenti \& Fischer 2005), whereas all stars from van Saders et al. (2016) are in a narrow mass range of $1.10 \pm 0.10 M_{\odot}$, see the bottom panel of Fig. 7. This difference suggests that the stars in the sample of van Saders et al. (2016) were originally F-type stars with higher initial mass and different internal structure than those of MW stars of similar age. The weak, if any, magnetic field 
of F-stars results in less effective magnetic braking that leaves the stars with faster rotation at advanced ages. The masses of the younger MW stars are on average about $1.0 M_{\odot} \pm 0.3$ with quite a large standard deviation.

\subsection{Wide binaries $H D 131156 \mathrm{~A} / \mathrm{B}$ and HD 201091/HD 201092 in the sample}

The stars of the wide binary system HD 131156A/B are the youngest stars (about $0.2 \mathrm{Gyr}$ ) of the MW stars in this paper, with fairly similar dominant cycles of about four years. The dominant cycles of the two components have amplitudes that are smaller than the long-term variability; the dominant cycles can therefore hardly be seen on the maps, which have no artificial amplifications (cf. Sect. 2). These dominant cycles are, however, evident in the time series themselves. Moreover, Morgenthaler et al. (2012) found from magnetic field monitoring of HD 131156A that the topologies of the field were similar in 2007.59 and 2011.07, that is, about $3.5 \mathrm{yr}$ apart, and suggested a possible relation with a cycle of this length, which supports our cycle length of $3.7 \mathrm{yr}$.

The stars of HD 201091/HD 201092 are near the VaughanPreston gap in the age- $P_{\text {cyc }} / P_{\text {rot }}$ diagram (Fig. 6, top panel), indicating slightly different evolutionary stages, possibly owing to the difference in mass between the stars $\left(0.690 / 0.605 M_{\odot}\right.$, Kervella et al. 2008). While HD 201091 has smooth cycles of about $7 \mathrm{yr}$, which slightly change with time, its later and less massive companion HD 201092 has a more erratic long-term variability (cf. Figs. 2 and 3). This different pattern is confirmed by the coronal variations of the stars. Robrade et al. (2012) used X-ray observations from XMM-Newton and sampled HD 201091 over the nine-year interval between 2002 and 2011. The authors confirmed the seven-year cycle found from the MW chromospheric record. HD 201092 showed irregular X-ray fluctuations around an intermediate state with slightly declining overall trend during the nine-year XMM observations that are well in line with those observed for chromospheric activity record.

\subsection{Place of the Sun among the cycling stars}

The Sun rotates slightly faster than the MW stars of similar ages, but except for this, the solar parameters and the results of the time-frequency analyses of solar datasets (sunspot number, Ca II K-line flux) fit the different relations drawn for the MW stars plotted in Figs. 4-6 well. One possible reason for the slightly faster rotation of the Sun in the sample is that according to Sellwood \& Binney (2002), the Sun is slightly metalrich compared with the stars of similar age, which may affect its rotational evolution because it makes magnetic braking less effective.

Another explanation for the slightly faster solar rotation might lie in the planetary system of the Sun. Recently, Maxted et al. (2015) found evidence that in some cases, host stars of exoplanets rotate faster than expected owing to their tidal spin-up. Apart from the Sun, only HD 3651 of the 29 MW stars we studied is known to have a planet, with an orbital period of 62.2 days on a very eccentric orbit $(e=0.63)$. It was discovered by Fischer et al. (2003) through radial velocity measurements. HD 3651 also has a distant T-dwarf companion star (e.g., Liu et al. 2007). The rotational period of HD 3651 is about 44 days, which is quite slow. Wittenmyer et al. (2013) showed that for HD 3651 (and for eight other cases outside the MW chromospheric activity samples) the radial velocity data can be better modeled with a planetary system of two planets on nearly circular orbits than with only one planet on an eccentric orbit. The second possible planet is closer to HD 3651, with a 31-day orbit. However, the two inferred planets are too small to affect the rotation of the central star.

\section{Summary}

We studied activity cycles of stars on or near the lower main sequence by analyzing observations of $29 \mathrm{MW}$ stars observed for $36 \mathrm{yr}$. The sample of stars covers a range of ages, with an average $\log g=4.5 \pm 0.17$. The older stars, older than 2-3 Gyr, form a homogeneous slowly rotating group of 12 stars with spectral types between $\mathrm{G} 5 \mathrm{~V}-\mathrm{K} 5 \mathrm{~V}$. On average, they have similar rotational periods of $39.7 \pm 6.0$ days. Their cycles look simple; the average dominant cycle length is $9.7 \pm 1.9 \mathrm{yr}$. In some cases, secondary low-amplitude cycles of about 3.5-4 yr also appear. The Sun itself rotates somewhat faster than the sample of 12 older stars with simple cycles. The universality of the sunspot Schwabe cycle of about $11 \mathrm{yr}$ for the Sun together with the average dominant cycle of about $10 \mathrm{yr}$ of the $12 \mathrm{MW}$ stars fits the recent popular attribution of the sunspot cycle well, which has been called "Nature's Third Cycle" by Choudhuri (2015).

In contrast, the younger 16 stars have a much faster rotation of $18.1 \pm 12.2$ days on average, with a large dispersion and shorter complex cycles of $7.6 \pm 4.9 \mathrm{yr}$, with additional sometimes multiple smaller amplitude cycles. The range of the spectral types for this group is somewhat wider (from F9.6V to K7V) than that of the older counterparts. There is a clear age separation between the two groups near the Vaughan-Preston gap (i.e., around 2-3 Gyr). This picture suggests that stars arriving at the lower main sequence are magnetically active and show fast rotation, high mean levels of magnetic activity, and complex interannual variations in activity. As they age, magnetic braking slows the rotation of the stars, leading to simpler cycling variations, similar to the contemporary solar Schwabe cycle.

The change in patterns we outlined may be due to a higher number of dynamo modes that are excited in the fast-rotating younger stars or to a more profound switch in the operation mode of the dynamo, as in a bimodal interface dynamo.

A clear implication of our results is that monitoring stellar activity variations is a rewarding and affordable investment, demonstrated by the presence of interannual-scale activity cycles. Observational efforts targeting activity cycles need at least a few decades to deliver reliable results, like the Mount Wilson project that spanned $36 \mathrm{yr}$, which, more than ten years after its termination, is still an extremely valuable source of information.

Acknowledgements. Thanks are due to the anonymous referee for the useful advice and suggestions. This work has been supported by the Hungarian Science Research Program OTKA-109276 and the Lendület-2012 Young Researchers' Programs of the Hungarian Academy of Sciences. K.O. is grateful to G. Kovács for enlightening discussions about stellar ages, and to A. Moór for useful advice. W.S. works were partially supported by SAO grant SAO-504514-421040504514SS5000 and SAO proposal 000000000003010-V101.

\section{References}

Baliunas, S. L., Donahue, R. A., Soon, W. H., et al. 1995, ApJ, 438, 269 Baliunas, S. L., Nesme-Ribes, E., Sokoloff, D., \& Soon, W. H. 1996, ApJ, 460, 848

Barnes, S. A. 2007, ApJ, 669, 1167

Barnes, S. A. 2010, ApJ, 722, 222

Barnes, S. A., Weingrill, J., Fritzewski, D., Strassmeier, K. G., \& Platais, I. 2016, ApJ, 823, 16

Barnhart, B. L., \& Eichinger, W. E. 2011, J. Atom. Solar-Terrestrial Phys., 73, 1771 
Bazilevskaya, G., Broomhall, A. M., Elsworth, Y., \& Nakariakov, V. M. 2014, Space Sci. Rev., 186, 359

Böhm-Vitense, E. 2007, ApJ, 657, 486

Brandenburg, A., Saar, S. H., \& Turpin, C. R. 1998, ApJ, 498, L51

Choudhuri, A. R. 2015, Nature's Third Cycle: A Story of Sunspots (New York: OUP), 293

Csubry, Z., \& Kolláth, Z. 1990, Proc. SOHO 14/GONG 2004 Workshop Helio- and Asteroseismology: Towards a Golden Future, 12-16 July, 2004. New Haven, Connecticut, USA, ed. D. Danesy, ESA SP, 559, 396

Donahue, R. A., Saar, S. H., \& Baliunas, S. L. 1996, ApJ, 466, 384

Durney, B. R., Mihalas, D., \& Robinson, R. D. 1981, PASP, 93, 537

Egeland, R., Metcalfe, T. S., Hall, J. C., \& Henry, G. W. 2015, ApJ, 812, 12

Favata, F., Micela, G., Orlando, S., et al. 2008, A\&A, 490, 1121

Fischer, D. A., Butler, R. P., Marcy, G. W., Vogt, S. S., \& Henry, G. W. 2003 , ApJ, 590, 1081

Forgács-Dajka, E., \& Borkovits, T. 2007, MNRAS, 374, 282

Gray, R. O., Corbally, C. J., Garrison, R. F., McFadden, M. T., \& Robinson, P. E. 2003, AJ, 126, 2048

Gray, R. O., Corbally, C. J., Garrison, R. F., et al. 2006, AJ, 132, 161

Kervella, P., Mérand, A., Pichon, B. et al. 2008, A\&A, 488, 667

Kolláth, Z. 1990, Konkoly Observatory Occasional Technical Notes, 1, 1

Kolláth, Z., \& Oláh, K. 2009, A\&A, 501, 695

Liu, M. C., Leggett, S. K., \& Chiu, K. 2007, ApJ, 660, 1507

Metcalfe, T. S., Buccino, A. P., Brown, B. P. et al. 2013, ApJ, 763, L26

McIntosh, S. W., Leamon, R. J., Krista, L. D., et al. 2015, Nature Comm, 6, 7491
Maxted, P. F. L., Serenelli, A. M., \& Southworth, J. 2015, A\&A, 577, A90

Meibom, S., Barnes, S. A., Platais, I., et al. 2015, Nature, 517, 589

Morgenthaler, A., Petit, P., \& Saar, S. 2012, A\&A, 540, A138

Noyes, R. W., Hartmann, L. W., Baliunas, S. L., Duncan, D. K., \& Vaughan, A. H. 1984, ApJ, 279, 763

Oláh, K., Kolláth, Z., Strassmeier, K. G., et al. 2009, A\&A, 501, 703

Pecaut, M. J. \& Mamajek, E. E. 2013, ApJS, 208, 9

Petrovay, K. 2007, Astron. Nachr., 328, 777

Petrovay, K., Kerekes, A., \& Erdélyi, R. 2010, Geophys. Astrophys. Fluid Dyn. 104, 619

Reiners, A., Schüssler, M., \& Passegger, V. M. 2014, ApJ, 794, 144

Robrade, J., Schmitt, J. H. M. M., \& Favata, F. 2012, A\&A, 543, A84

Roskar, R., Debattista, V. P., Quinn, T. R., \& Wadsley, J. 2012, MNRAS, 426, 2089

Sellwood, J. A., \& Binney, J. J. 2002, MNRAS, 336, 785

Soon, W. H., Baliunas, S. L., \& Zhang, Q. 1993a, ApJ, 414, L33

Soon, W. H., Zhang, Q., Baliunas, S. L., \& Kurucz, R. L. 1993b, ApJ, 416, 787

Soon, W., Velasco Herrera, V. M., Selvaraj, K., et al. 2014, ESR 134, 1

Valenti, J. A., \& Fischer, D. A. 2005, ApJS 159, 441

van Saders, J., Ceillier, T., Metcalfe, T., et al. 2016, Nature, 529, 181

Vaughan, A., \& Preston, G. W. 1980, PASP, 92, 385

Wilson, O. C. 1968, ApJ, 153, 221

Wilson, O. C. 1978, ApJ, 226, 379

Wittenmyer, R. A., Wang, S., Horner, J. et al. 2013, ApJS, 208, 2 


\section{Appendix A: Additional figures}
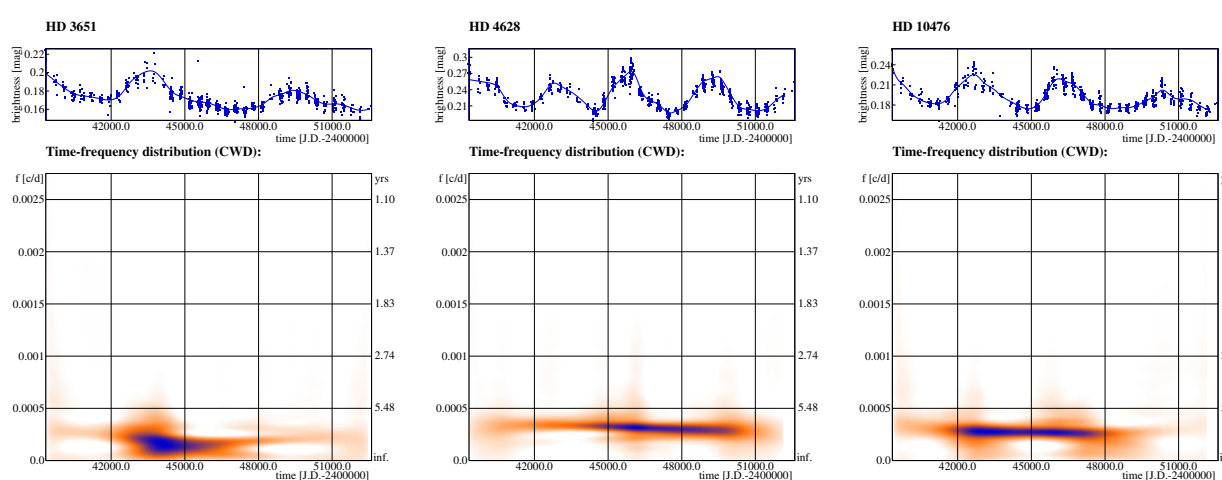

HD 16160

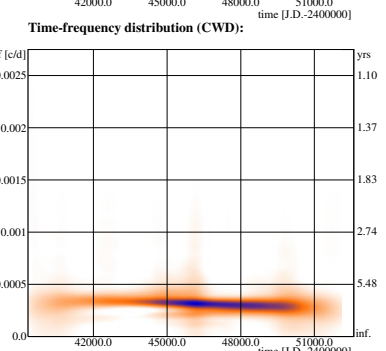

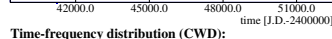

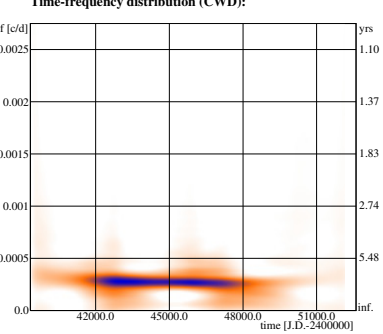

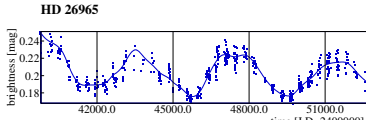

$$
\text { HD } 32147
$$

HD 81809
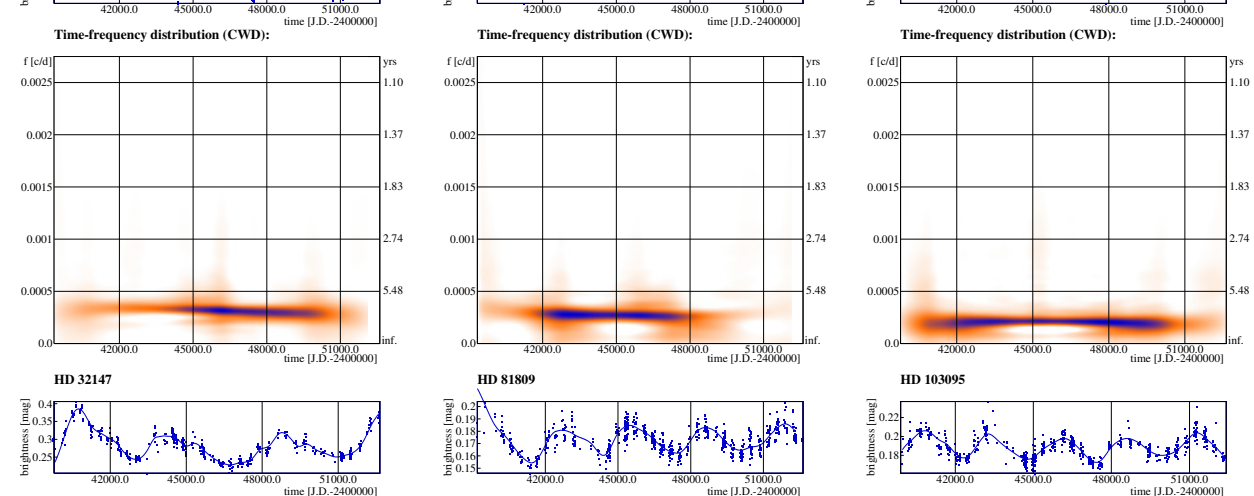

Time-frequency distribution (CWD):

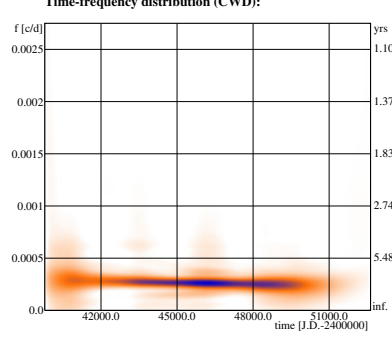

HD 160346

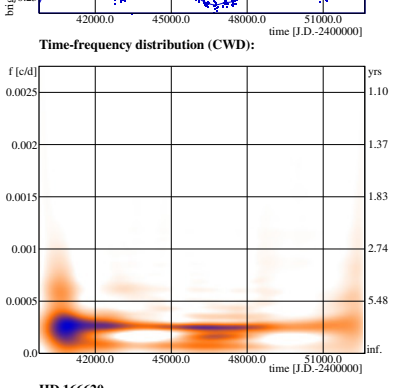

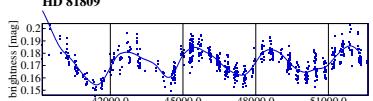

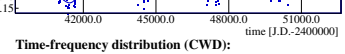

HD 103095
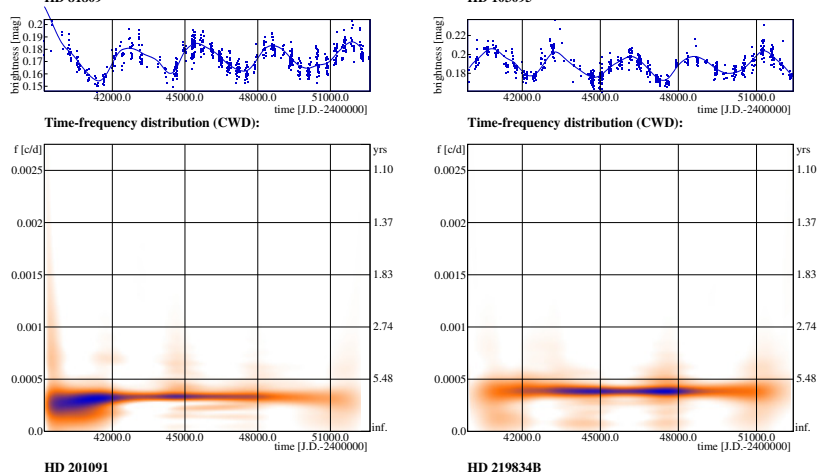

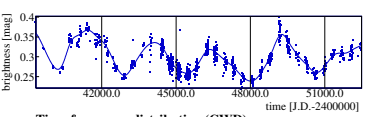
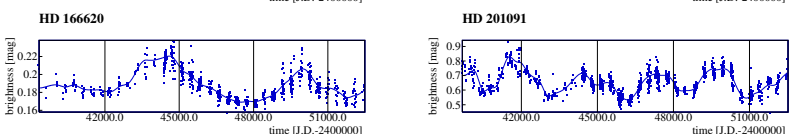

HD 219834B

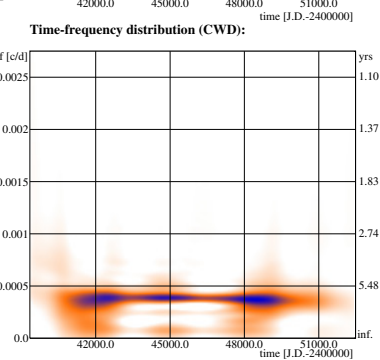

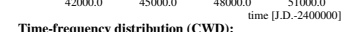
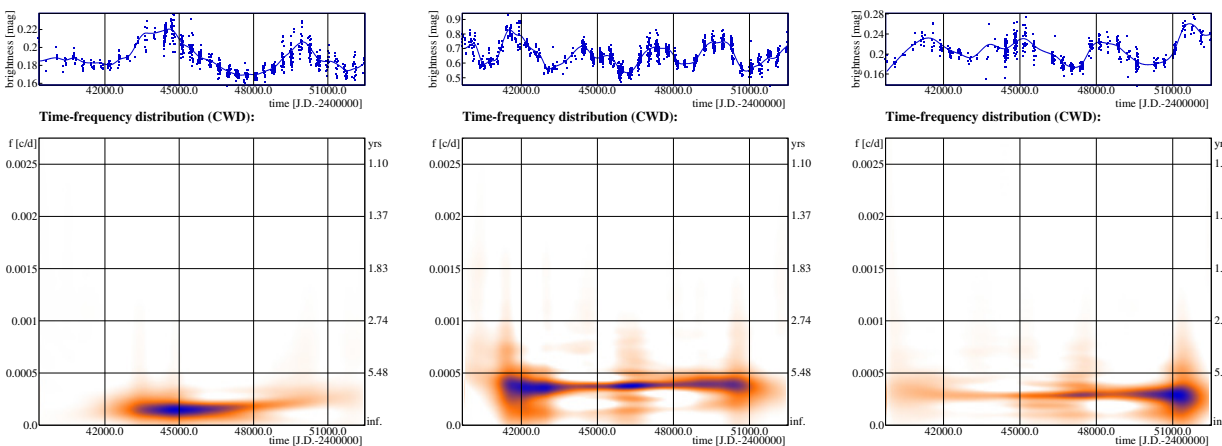

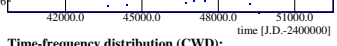

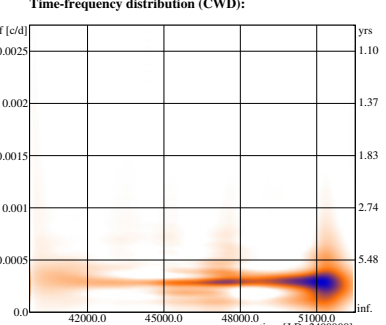

Fig. A.1. CWD of the old MW stars with simple cycles. Upper panels: datasets and the spline interpolation; Lower panels: time-frequency diagrams. 

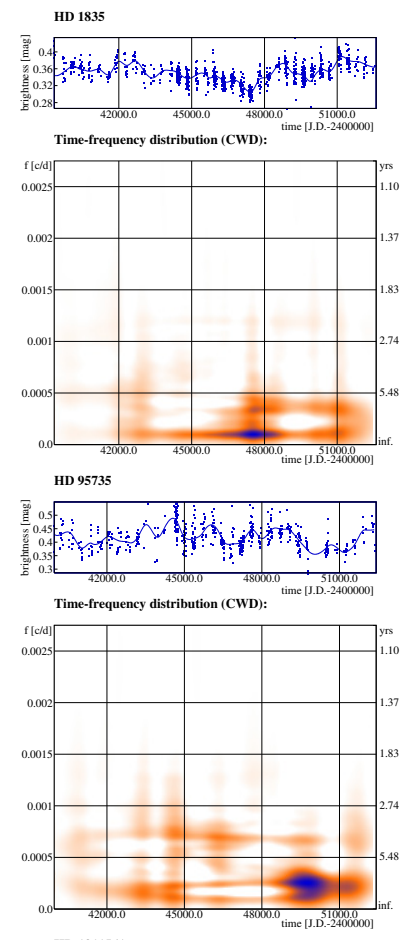

HD 131156A
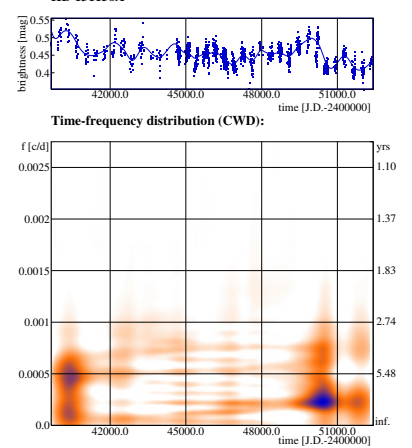

$$
\text { HD } 156026
$$
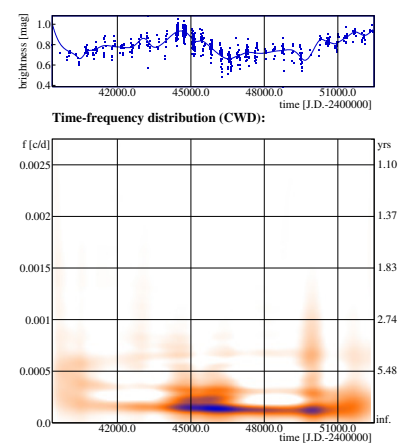

HD 201092
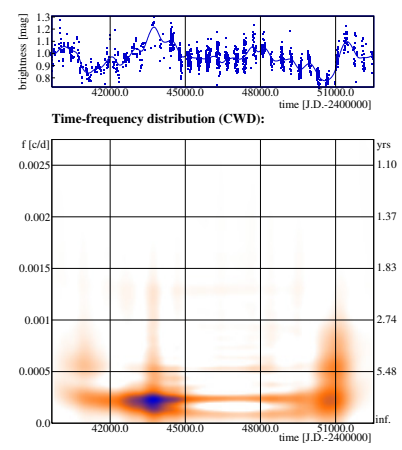

HD 20630

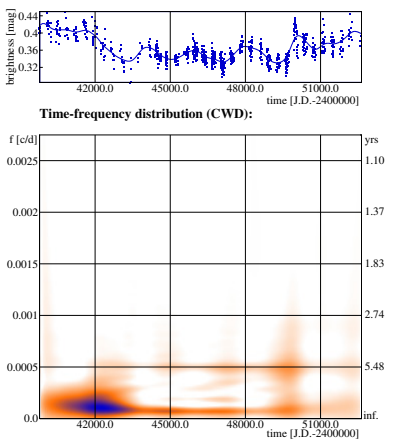

HD 100180

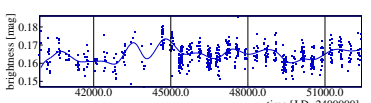
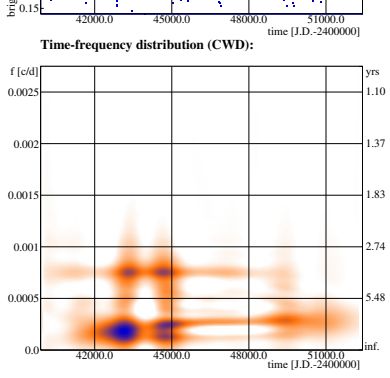

HD 131156B

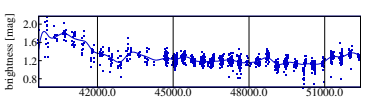

420100.0
450000.0
Time-frequency distribution (CWD):

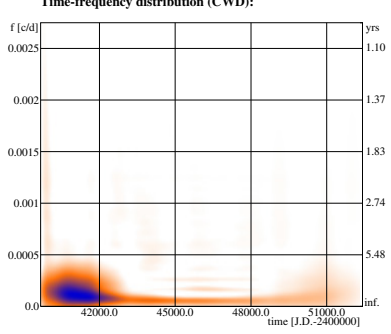

HD $165341 \mathrm{~A}$

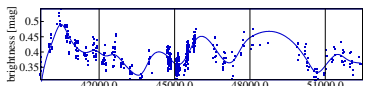

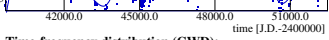

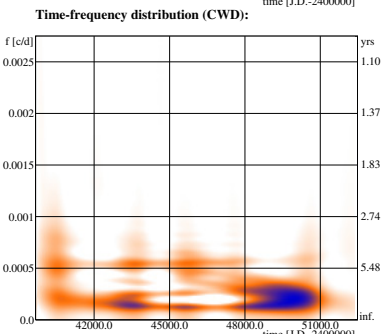

HD 76151

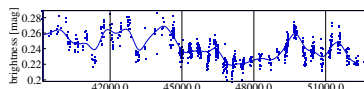

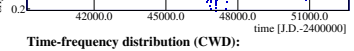

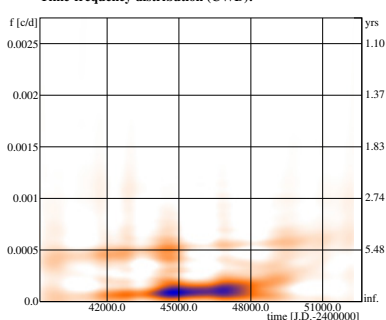

HD 114710

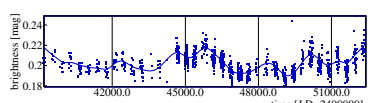

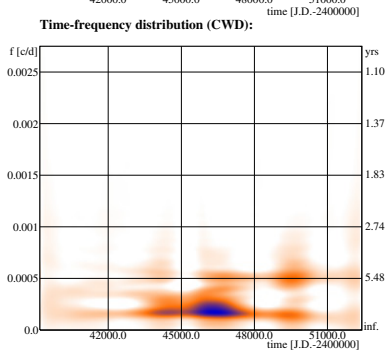

HD 149661

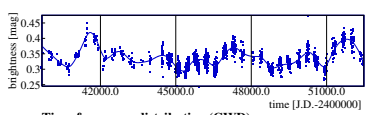

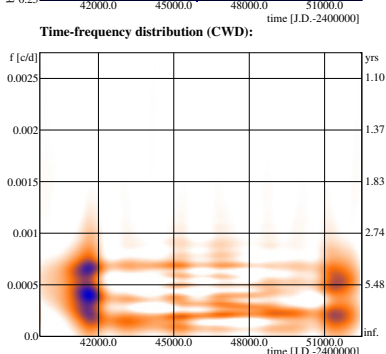

HD $165341 \mathrm{~B}$

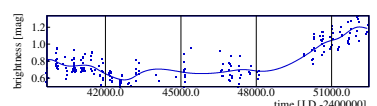

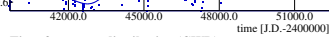

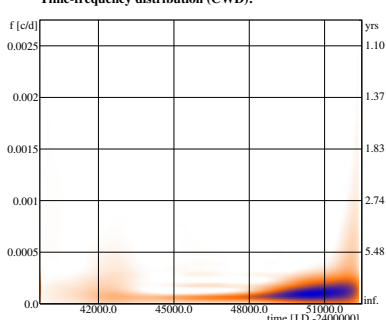

HD 78366

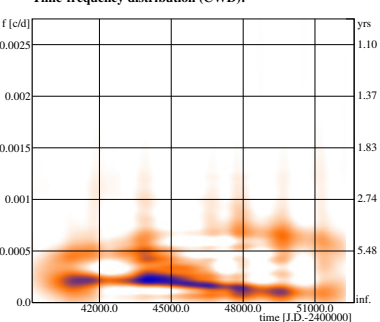

HD 115404

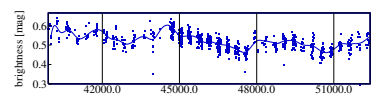

420100.00
Time-frequency distribution (CWD):

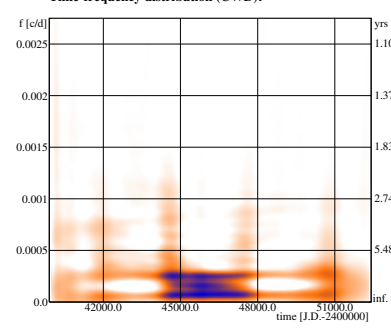

HD 15239

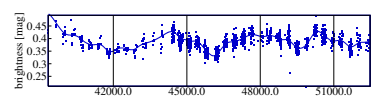

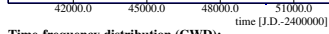

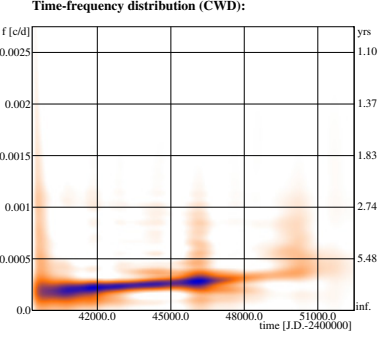

HD 190406

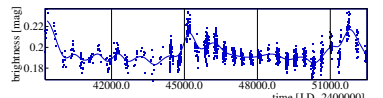

420100.0
Time-frequency distribution (CWD):

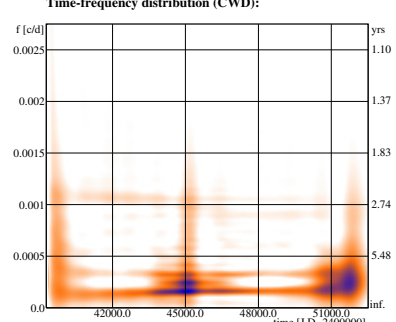

Fig. A.2. CWD of the young MW stars with complex cycles. Upper panels: datasets and the used spline interpolation; lower panels: time-frequency diagrams. 
K. Oláh et al.: Magnetic cycles at different ages of stars

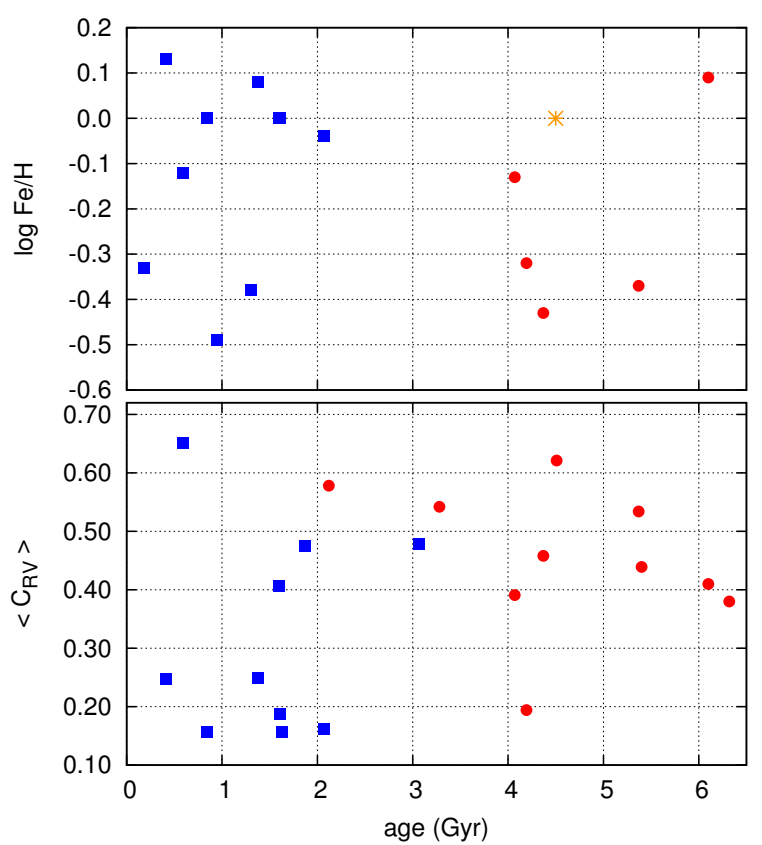

Fig. A.3. Metallicity vs. age of the MW stars. Top: $\log (\mathrm{Fe} / \mathrm{H})$ from Gray et al. (2003, 2006), bottom: $\left\langle C_{R V}\right\rangle$ from Soon et al. (1993b). 